\title{
DEVELOPING RE-USABLE INTERACTIVE STORYTELLING TECHNOLOGIES
}

\author{
Marc Cavazza, Fred Charles and Steven J. Mead \\ School of Computing, University of Teesside, TS1 3BA, Middlesbrough, UK \\ (http://www-scm.tees.ac.uk/virtualstorytelling) \\ \{m.o.cavazza,f,charles, steven.j.mead\}@tees.ac.uk
}

\begin{abstract}
Despite the growing interest in Interactive Storytelling (IS), there have been only a small number of implemented demonstrators and few have attempted at developing a re-usable IS technology. In this paper we describe such an IS engine, which is the result of several years of experimentation in the field. The system is based on a game engine for its visualisation component, while the narrative generation component implements a variant of HTN Planning. After an introduction to the principles underlying the system, we introduce the associated production process and discuss authoring problems as well as tools we have developed to facilitate the use of the technology.
\end{abstract}

Key words: Interactive Narratives, Virtual Storytelling, Artificial Actors.

\section{INTRODUCTION AND STATE OF THE ART}

One of the most ambitious aspects of developing Interactive Storytelling (IS) as a new medium consists in establishing technologies supporting interactive narratives. With the recent development of IS, there is now an emerging consensus among those teams who have developed substantial IS prototypes [Cavazza et al., 2002] [Mateas and Stern, 2002] [Swartout et al., 2001] [Young, 2001] on which computing technologies are central to that endeavour. IS technologies should support the real-time presentation of a story whose plot is generated dynamically, so as to accommodate the result of user intervention. At the same time, the existence of narrative formalisms (plot-based or character-based) guarantees plot coherence and provide practical solutions to the narrative control problem. Both aspects, dynamic 
generation of situations and the representation of a story baseline, can be addressed using Artificial Intelligence techniques. From a formal perspective the backbone of the narrative is constituted by a sequence of actions: it hence comes as no surprise that AI planning is considered by many as a central technology for IS [Cavazza et al., 2002] [Young, 2001]. However, attention should also be paid to the representation component of IS technologies, as it conditions the authoring of the story baseline which should play an essential role in plot coherence and narrative control.

As a result of several years of experimenting with these issues, we have produced various research prototypes, which recently have demonstrated some potential for scalability. In this paper we are presenting the IS engine we have developed as a technology which, even if far from being mature, can be shared among the research community.

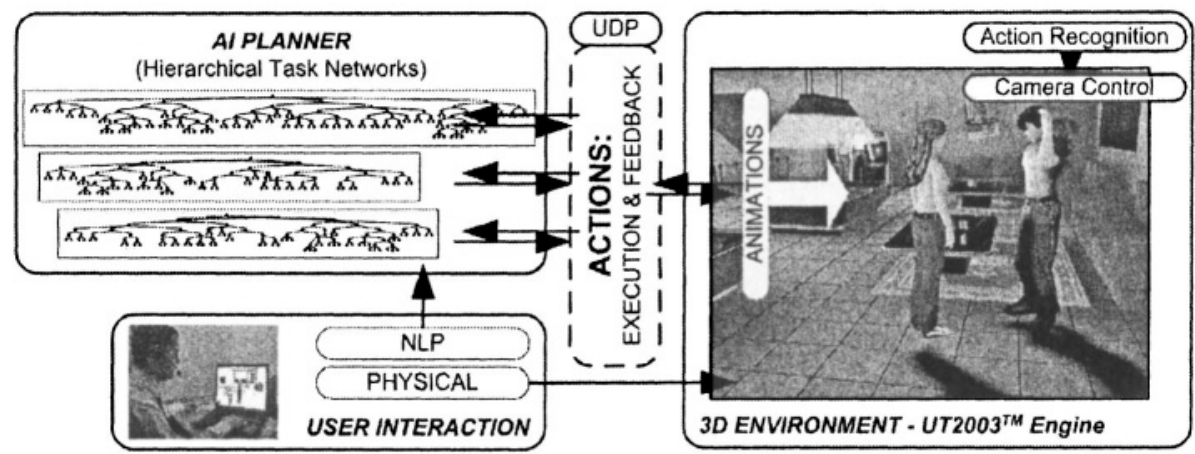

Figure 1. System overview.

\section{SYSTEM ARCHITECTURE}

Our technology (Figure 1) is developed on top of a computer game engine (UT 2003 ${ }^{\mathrm{TM}}$, Epic Games), which is an approach inspired from foundational work in IS [Young, 1999] [Young, 2000]. The game engine ensures real-time visualisation (including camera control) as well as basic interaction mechanisms (between agents, between agents and objects, etc.). We have developed several additional layers (amounting to a total of 10000 lines of $\mathrm{C}++$ code and 8000 lines of UnrealScript code) corresponding to the narrative engine, which is essentially a HTN planner determining for each virtual actor what action it should take next. The actions selected are passed to the engine, in which they are associated to corresponding scripts describing the physical realisation of the action (including specific animations). The planner communicates with the visualisation engine using UDP socket connections. The two mechanisms for story generation are interaction between agents and user intervention. The latter is essentially 
mediated by speech input, which is why the system accepts data from an offthe-shelf speech recognition system. The recognised utterance is further processed into templates that can be interpreted in terms of narrative content.

\section{STORY GENERATION AND PERFORMANCE}

To a large extent, the system's approach has been generalised from the study of specific story genres, such as the sitcom genre. More recent experiments with another narrative genre would suggest that the system is not strongly biased towards its early supporting examples; however, it is likely that some assumptions such as task decomposability would fail to properly represent certain kind of narratives. Even within the subset of genres for which the approach is appropriate, system performance should be judged by the system's ability to generate relevant (ideally, interesting) stories and by its potential for scalability. The whole field still lacks metrics for the evaluation of a story's narrative qualities. This is why a basic measure of story generation consists in recording beats [Mateas and Stern, 2002], as a minimal (semantic) unit of narrative action, probably assimilable to a narrative function. Mateas and Stern have proposed an evaluation metric for the maturity of IS techniques, which consisted in being able to generate a 10-minute story comprising at least one beat per minute.
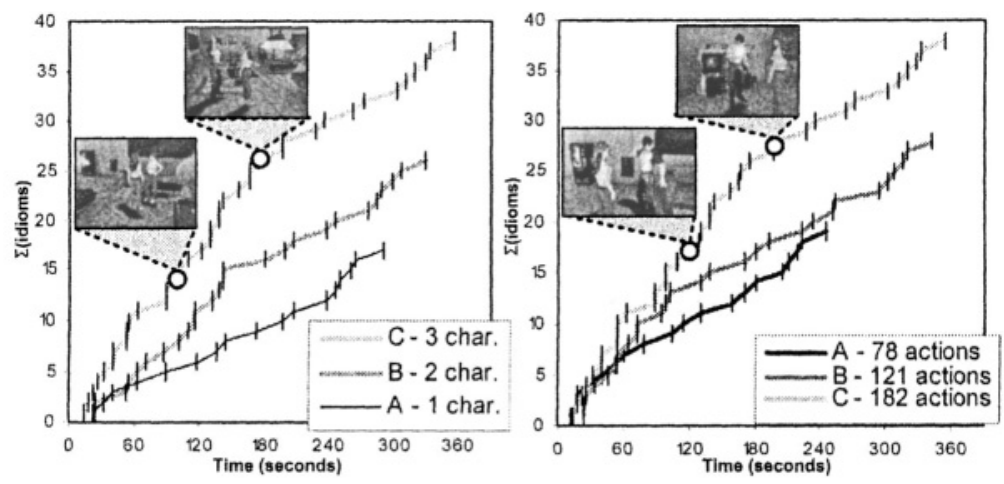

Figure 2. Scalability of character-based storytelling.

We have conducted several scalability experiments demonstrating the increase in story sophistication as the individual characters roles are made more complex [Charles and Cavazza, 2003]. Figure 2 shows the increase in story duration and number of situations generated (measured through film idioms, a formalisation of situations used in Film Studies) for various versions of a same story, each version differing by the complexity of individual characters' roles. 


\section{THE DEVELOPMENT PROCESS}

The creation of an interactive narrative with our system proceeds through several steps which are summarised on Figure 3. Once again it is helpful to keep in mind the distinction between story generation and presentation; in other words, generating the sequence of actions and for each of these actions, staging them in real-time in the most appropriate fashion.

The first aspect consists in creating all the visual elements for the interactive story: the virtual stage can be developed as a UT $2003^{\mathrm{TM}}$ level for which the editor provides sophisticated features. The 3D contents can be imported from popular modelling packages and the textures can be generated from various image formats, apart from an extensive range existing in the system's own library (we do not discuss character's animations as these are related to the definition of primitive actions in the next section).

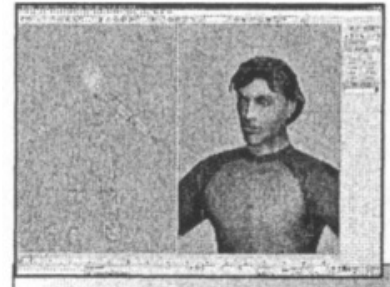

Modelling, Animation, Texturing

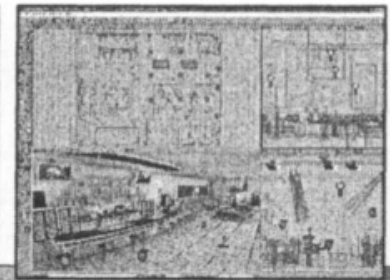

3D Environment integration (Univaliegl.

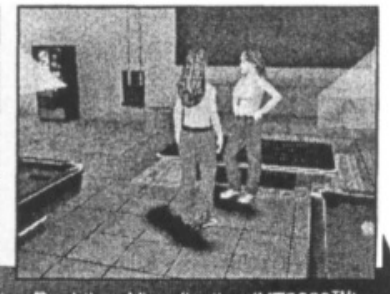

Real-fime Visualisation (UT2003 ${ }^{\mathrm{TM}}$ )

Figure 3. Integration of creative contents in the virtual environment.

Probably the most central part of authoring an IS system is to instantiate the knowledge representation underlying the story generation system. In our approach, this consists in formalising the role of each character into an HTN. This is done by identifying various phases of the character's role, then expressing them as a decomposition of tasks into sub-tasks. Because we have developed a specific graphic interface for that purpose, we will describe this process in the next section.

The generation of actions for each character is based on a role formalised as an HTN, which decomposes the role into independent tasks, ordered chronologically, until these tasks can be solved through elementary actions. The system relies on the native mechanisms of UT $2003^{\mathrm{TM}}$ for many of these actions (such as path planning, walking, grabbing objects, etc.). However, once terminal actions have been identified a script should be generated for its enactment encapsulating all relevant system primitives. As for the visual presentation, many actions require specific animations to be imported into the system (actions such as sitting, or kissing, are obviously not part of the native library of a first-person shooting game).

In our character-based approach, it is not possible (and not even desirable) to incorporate within each single character's role an anticipation 
of all possible situations that can arise dynamically from the interaction between characters. Hence it can be necessary to complement the top-down approach with the bottom-up description of situated reasoning that would react to such situation.

Finally, as spoken utterances are the most appropriate interface for user interventions in the story [Cavazza et al., 2002] [Mateas and Stern, 2002], there is a need to author these aspects as well. However this kind of authoring depends on the specific speech recognition component adopted, which is why we shall not discuss it here.

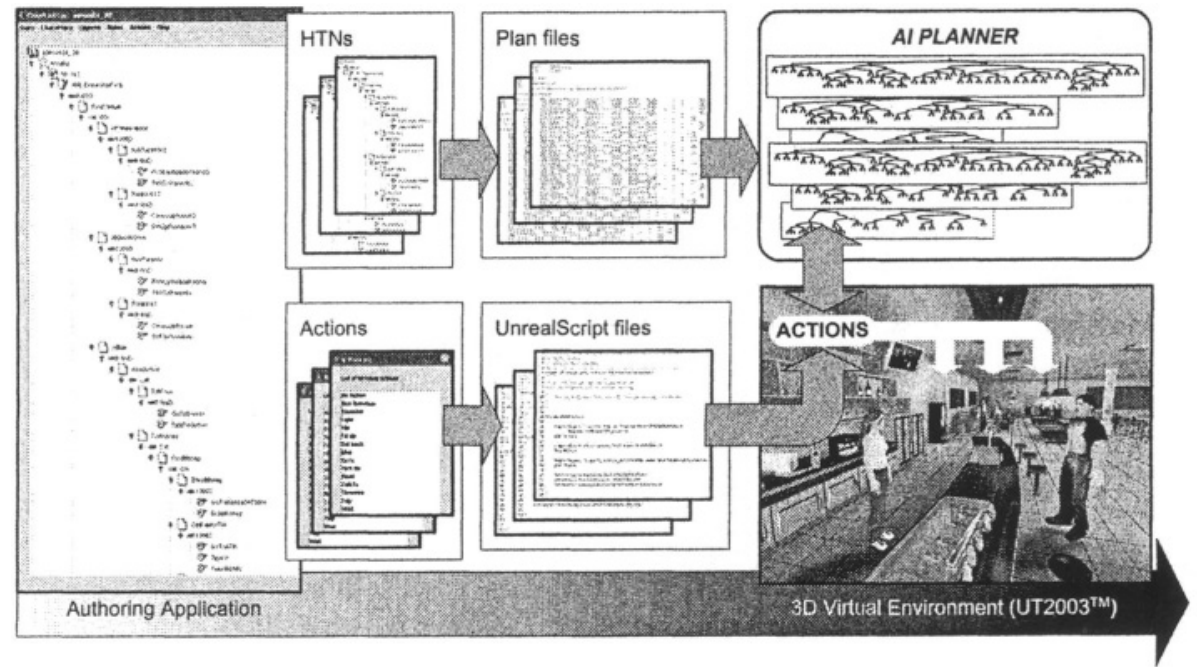

Figure 4. From story authoring to dynamic story generation.

\section{THE AUTHORING INTERFACE}

One of the limiting factors of the development of IS is likely to be the difficulty to author interactive narratives. Despite the dynamic nature of such stories, whose generative aspects depend not only from user intervention but also from internal interactions between the story characters, the value of the baseline narrative still plays an essential role in underlying the overall consistency of the narrative. At this early stage it remains unclear whether interactive stories will be created by traditional scriptwriters or by a new breed of story designers, closer to those creating computer games. In order to remain as neutral as possible on this issue, we have devised a user interface supporting the authoring of characters' roles in our IS engine. This interface does not address the whole content production chain as discussed above, but is centred on its most technical aspect, which is the definition of role-plans for the IS characters (Figure 4). We have previously discussed the authoring 
problems associated with planning formalisms in IS [Cavazza et al., 2002], and have concluded that one of the benefits of HTN formalisms are their visual and integrated nature. We hypothesised that this visual nature should facilitate authoring by content creators, who would proceed by incremental task decomposition when describing a character's role, relying on the intuitive semantics of the formalism.

\section{CONCLUSIONS}

We have described a fully implemented IS prototype, for which we claim a possible re-use of its underlying technology. We have identified several key steps in the production process and have developed user-friendly interfaces to facilitate some aspects of authoring identified as critical for the use of the technology.

We have only recently started to distribute alpha versions of the system, which have been used for teaching AI-based animation and multi-agent systems at our institution, as well as others. Despite only implementing one possible paradigm of IS, we hope it could be useful, not only to researchers in IS but also in related areas such as training and edutainment, which can develop applications on top of these technologies.

\section{Acknowledgements}

We would like to give credits to the designers of the character models, Demiurge Studios.

\section{References}

Cavazza M, Charles F, Mead SJ. Character-based Interactive Storytelling”, IEEE Intelligent Systems, 17(4), 2002.

Charles F and Cavazza M. Exploring the Scalability of Character-based Storytelling. ACM Joint Conference on Autonomous Agents and Multi-Agent Systems, New York, USA, 2004 (to appear).

Mateas M, Stern A. A Behavior Language for Story-based Believable Agents", IEEE Intelligent Systems, 17(4), 2002.

Swartout W, Hill R, Gratch J, Johnson WL, Kyriakakis C, LaBore C, Lindheim R, Marsella S, Miraglia D, Moore B, Morie J, Rickel J, Thiebaux M, Tuch L, Whitney R, Douglas J. Toward the Holodeck: Integrating Graphics, Sound, Character and Story. Proceedings of the Autonomous Agents 2001 Conference; Montreal, Canada, 2001.

Young RM. Notes on the Use of Plan Structures in the Creation of Interactive Plot. AAAI Fall Symposium on Narrative Intelligence. AAAI Press, 1999.

Young RM. Creating Interactive Narrative Structures: The Potential for AI Approaches.

AAAI Spring Symposium in Artificial Intelligence and Entertainment. AAAI Press, 2000.

Young RM, An Overview of the Mimesis Architecture: Integrating Intelligent Narrative Control into an Existing Gaming Environment, Working Notes of the AAAI Spring Symposium on Artificial Intelligence and Interactive Entertainment, AAAI Press, 2001. 